\title{
Importance of atrial surface area and refractory period in sustaining atrial fibrillation: Testing the critical mass hypothesis
}

\author{
Anson M. Lee, MD, Abdulhameed Aziz, MD, Jacob Didesch, BS, Kal L. Clark, MD, \\ Richard B. Schuessler, PhD, and Ralph J. Damiano, Jr, MD
}

\begin{abstract}
Objective: The critical mass hypothesis for atrial fibrillation (AF) was proposed in 1914; however, there have been few studies defining the relationship between atrial surface area and AF. This study evaluated the effect of tissue area and effective refractory period (ERP) on the probability of sustaining AF in an in vivo model.
\end{abstract}

\begin{abstract}
Methods: Domestic pigs $(\mathrm{n}=9)$ underwent median sternotomy. Epicardial activation maps were constructed from bipolar electrograms recorded from form-fitting electrode templates placed on the atria. Baseline ERPs were determined. ERP was lowered with a continuous infusion of acetylcholine $(0.005-0.04 \mathrm{mg} / \mathrm{Kg} / \mathrm{min})$ until AF could be sustained after burst pacing. The atria were sequentially partitioned using bipolar radiofrequency ablation. ERPs were lowered using acetylcholine until AF could be sustained in each subdivision of atrial tissue. Each subdivision was further divided until AF was no longer inducible. At study completion, the heart was excised and the surface area of each section was measured.
\end{abstract}

Results: Over a range of ERPs from 75 to $250 \mathrm{~ms}$, the probability of AF was correlated with increasing tissue area (range, $\left.19.5-105 \mathrm{~cm}^{2}\right)$ and decreasing ERP. Logistic regression analysis identified shorter ERP $(P<.001)$ and larger area $(P=.006)$ as factors predictive of an increased probability of sustained $\mathrm{AF}$ (area under the curve of the receiver-operator characteristic $=0.878$ ).

Conclusions: The probability of sustained AF was significantly associated with increasing tissue area and decreasing ERP. These data may lead to a greater understanding of the mechanism of AF and help to design better interventional procedures. (J Thorac Cardiovasc Surg 2013;146:593-8)

Atrial fibrillation (AF) remains a significant clinical problem and is the most common sustained arrhythmia in the United States, affecting over 2 million Americans. ${ }^{1}$ It is a significant cause of morbidity and is thought to be responsible for $15 \%$ to $20 \%$ of all strokes. ${ }^{1}$ In a population-based study, AF has been shown to be an independent risk for increased mortality. ${ }^{2}$ Unfortunately, the mechanism underlying AF remains poorly understood.

In 1914 , Garrey ${ }^{3}$ hypothesized that a critical mass of atrial tissue was necessary to sustain AF. He theorized that multiple wavelets of electrical activity propagated through the atrium, activating it in an unorganized fashion, thus leading to fibrillation. Later, Weiner and Rosenbluth ${ }^{4}$

\footnotetext{
From the Division of Cardiothoracic Surgery, Department of Surgery, Washington University School of Medicine, St Louis, Barnes-Jewish Hospital, St Louis, Mo. This study was supported in part by National Institutes of Health grants 5R01 HL032257, R01 HL085113, and T32 HL07776. R.B.S. has received research grants from AtriCure, Inc (West Chester, Ohio) and Estech, Inc (San Ramon, Calif). R.J.D. has received research grants from AtriCure, Inc, Estech, Inc, and Medtronic, Inc (Minneapolis, Minn). R.J.D. receives consultant fees from AtriCure, Inc, and Medtronic, Inc.

Disclosures: Authors have nothing to disclose with regard to commercial support. Received for publication Oct 13, 2011; revisions received March 20, 2012; accepted for publication April 6, 2012; available ahead of print Sept 19, 2012.

Address for reprints: Ralph J. Damiano, Jr, MD, Department of Cardiac Surgery, Washington University School of Medicine, St Louis, Barnes-Jewish Hospital, Campus Box 8234, 660 S Euclid Ave, St Louis, MO 63110 (E-mail: damianor@ wustl.edu).

0022-5223/ $\$ 36.00$

Copyright (C) 2013 by The American Association for Thoracic Surgery http://dx.doi.org/10.1016/j.jtcvs.2012.04.021
}

introduced the concept of wavelength. Defined as the product of conduction velocity and refractory period, it is the minimum path length necessary for reentry in sustained AF. More recently, computer simulations of atrial sheets have demonstrated that the probability of fibrillation is dependent on increasing surface area. ${ }^{5,6}$ Our laboratory has demonstrated the validity of the critical mass hypothesis in isolated canine atria. ${ }^{7}$

It is now understood that the mechanisms underlying $\mathrm{AF}$ are more complex and varied than multiple wavelets. AF may be the result of triggered activity, most commonly originating from the pulmonary veins. ${ }^{8}$ It may also be the result of multiple wavelets of reentry as originally hypothesized, or it may be the result of a stable single rotor of reentrant activity that conducts in a fibrillatory manner. ${ }^{9}$ Despite the complexity underlying the mechanisms of AF, successful procedures have been designed and implemented on the basis of this incomplete understanding. The Cox maze procedure was designed as an empirical operation to interrupt all possible reentrant circuits in AF by making incisions in both atria to create lines of conduction block. ${ }^{10}$ This procedure has been very successful in treating $\mathrm{AF}$, with success rates near $80 \%$ in preventing recurrent $\mathrm{AF}$ without antiarrhythmic drugs in a recent report. ${ }^{11}$

There remains a subset of patients for which the Cox maze procedure is not effective. Patients with large left atria are known to be susceptible to recurrent $\mathrm{AF}^{12}$ It is hypothesized that for patients with recurrent $\mathrm{AF}$, the Cox maze 


\section{Abbreviations and Acronyms \\ $\mathrm{AF}=$ atrial fibrillation \\ $\mathrm{CT}=$ computed tomography \\ $\mathrm{ECG}=$ electrocardiogram \\ $\mathrm{ERP}=$ effective refractory period}

procedure does not divide the atria into small enough sections to prevent sustained $\mathrm{AF}$.

Work from this laboratory demonstrated the effects of atrial surface area and changing effective refractory period (ERP) on the sustainability of AF in an in vitro model. ${ }^{7}$ In isolated canine atria, larger atrial surfaces areas and shorter ERPs were associated with a high probability of sustained $\mathrm{AF}$ as predicted by the critical mass hypothesis. However, this has not been demonstrated in an intact animal model. The goal of this study was to evaluate the effect of tissue area, conduction velocity, and ERP on the sustainability of $\mathrm{AF}$ in an in vivo porcine model.

\section{METHODS}

\section{Acetylcholine Dose Response}

A preliminary study was conducted to establish the dose-response relationship between the ERP and acetylcholine systemically infused in the intact porcine model. The ERP was measured periodically during a continuous infusion of acetylcholine and for a period after acetylcholine was discontinued to establish typical recovery time for measured ERP. Three domestic pigs weighing 70 to $85 \mathrm{~kg}$ were used for this initial experiment. All animals received humane care in compliance with the "Guide for the Care and Use of Laboratory Animals" (National Academy Press, Washington, DC). Each animal was premedicated with tiletamine/zolazepam (Telazol), ketamine, and xylazine, intubated, anesthetized with isoflourane, and monitored continuously throughout the procedure with electrocardiographic (ECG) and invasive arterial pressure recordings.

A median sternotomy was performed and a pericardial sling was created. Two bipolar electrodes were sutured to the right atrium. One bipolar electrode was used for pacing, and another bipolar electrode was used to record atrial electrograms. Baseline pacing thresholds were measured at the beginning of each experiment, and subsequent pacing was conducted at twice the pacing threshold. Eight stimuli with a basic cycle length of $300 \mathrm{~ms}$ (S1) followed by a single extrastimulus was used with variable cycle length (S2) were used to obtain ERP.

After baseline ERP was measured, a continuous infusion of acetylcholine was initiated through a 20-gauge catheter inserted into the left atrium through a purse-string suture. An initial dose of $0.005 \mathrm{mg} / \mathrm{Kg} / \mathrm{min}$ was chosen on the basis of prior studies in dogs from the literature. ${ }^{13}$ Infusion was continued for 20 minutes. ERP was measured at time 0 and at every 5 minutes during the infusion and recovery period on the right and left atria. After 10 minutes of stable sinus rhythm after infusion of acetylcholine, the dose of acetylcholine was increased and the process was repeated. This was repeated for doses of $0.005,0.01,0.015$, and $0.02 \mathrm{mg} / \mathrm{Kg} / \mathrm{min}$. Occasionally, the atria would fibrillate. If this happened, the atria were allowed to fibrillate for 1 minute, after which an electrical cardioversion was performed.

\section{Critical Mass Study}

Nine domestic pigs weighing 60 to $85 \mathrm{~kg}$ were studied. Each animal was premedicated with tiletamine/zolazepam, ketamine, and xylazine, intubated, and anesthetized with isoflurane. The ECG and arterial pressure recordings were continuously monitored. Blood gases and electrolytes were determined and normalized every 20 minutes throughout the study.

Each animal had a set of atrial ablations performed through a median sternotomy (Figure 1). An iterative approach was used to induce AF. Before ablation, 2 bipolar pacing electrodes were sutured onto the right and left atria. Baseline ERP was measured using the single extrastimulus pacing technique. After ERP was measured, an attempt was made to induce AF by burst pacing. If necessary, a continuous infusion of acetylcholine was used to lower ERP until AF could be sustained. A set of 3 molded silicone plaques with a total of 252 unipolar electrodes were placed onto the epicardial surface to obtain epicardial electrograms, which were used to construct activation sequence maps. The electrode templates were constructed from a form-fitting silicone elastomer (Specialty Silicone Fabricators, Paso Robles, Calif) and contained 0.5-mm diameter silver electrodes (Pacific Wire \& Cable, Inc, Santa Ana, Calif). The interelectrode distance was $5 \mathrm{~mm}$. These plaques were secured with Rommel tourniquets to allow for consistent placement before and after each ablation. Electrograms were acquired during normal sinus rhythm, paced rhythm at 180 beats/min, and for at least 10 seconds during each episode of $\mathrm{AF}$ and used for offline analysis after each study. ${ }^{7}$ Data were recorded at a gain of 125 and frequency response of 0.5 to $500 \mathrm{~Hz}$ and digitized at $1000 \mathrm{~Hz}$. Activation times were calculated by determining the maximum negative instantaneous rate of voltage change over time. Activation maps were displayed on a 3-dimensional model of the atrial surface. Conduction velocity was calculated from the epicardial activation maps during paced rhythm. Next, the atria were subdivided with transmural linear ablations created by a bipolar radiofrequency ablation device (Isolator Atricure, Cincinnati, Ohio) (Figure 1). The process of measuring ERP and inducing AF was repeated in each subdivision until AF could no longer be sustained despite maximal doses of acetylcholine. Minimal ERP in each subdivision of atrial tissue was estimated using the minimal AF cycle length from the electrogram recordings for each instance of AF. It has been shown that this method accurately estimated the ERP during $\mathrm{AF}$ in an isolated canine atrial model. ${ }^{14}$ During episodes of AF, these estimated minimal ERPs were used in the analysis for the study. This was done to obtain the minimal ERP in a given tissue section.

When the atrium did not fibrillate, the ERP was measured directly. To measure ERP, we determined pacing thresholds from each of the pacing electrodes. Subsequent pacing was carried out at twice threshold. After each ablation, thresholds were remeasured. Eight stimuli with cycle lengths of $300 \mathrm{~ms}$ (S1) followed by a single extrastimulus was used with variable cycle lengths (S2) used to manually obtain ERP. To induce AF in the instances in which a single extra beat did not induce AF, we used burst pacing with a cycle length set to $45 \mathrm{~ms}$. Burst pacing continued for 30 seconds, and sustained AF was defined as AF that lasted longer than 30 seconds.

If the section of atrium failed to sustain AF after 2 attempts, a continuous infusion of acetylcholine was used to lower the ERP of the atria. A catheter was placed through a purse-string suture directly into the left atrium to minimize the exposure of the drug to cholinesterases before reaching the coronary circulation. The starting dose was $0.005 \mathrm{mg} / \mathrm{Kg} / \mathrm{min}$ and was increased in increments of $0.005 \mathrm{mg} / \mathrm{Kg} / \mathrm{min}$ to a maximum dose of 0.04 $\mathrm{mg} / \mathrm{Kg} / \mathrm{min}$. Acetylcholine was infused for 5 minutes before measurements were begun to allow for the ERP to stabilize. During infusion of acetylcholine, intravenous infusion of phenylepherine $(1-2 \mathrm{mg} / \mathrm{kg} / \mathrm{min})$ was used to maintain a mean blood pressure above $50 \mathrm{~mm} \mathrm{Hg}$.

The lesion set to subdivide the atrium is shown in Figure 1. All ablations were performed with a bipolar radiofrequency clamp device. This technology has been shown to reliably create transmural lesions and bidirectional conduction block and at the same time preserve the circulation to the atrial tissue. ${ }^{15}$ An initial ablation was performed to encircle a cuff of atrial tissue around the left and right pulmonary veins, respectively. The atrial surface area outside these ablation lines served as the baseline surface area for induction of AF. The first division of atrial surface area was obtained by creating a line of ablation extending from the left pulmonary vein ablation 


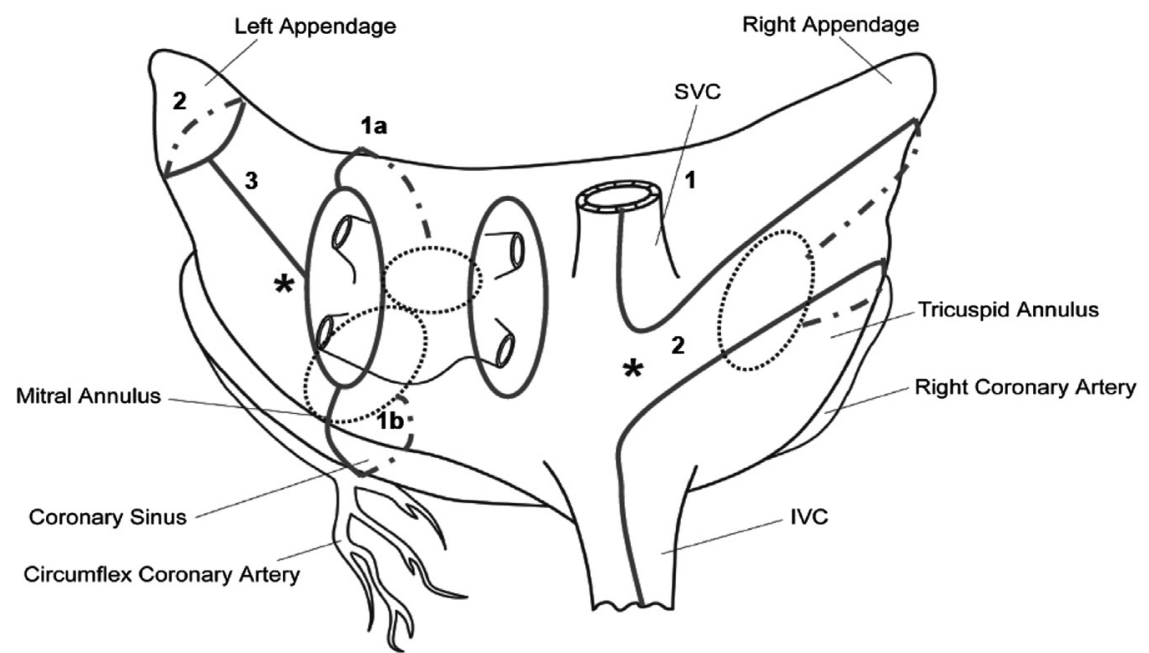

FIGURE 1. The lesion set used to subdivide the atrium. After ablation around the pulmonary veins as a starting point, the atria were divided into 2 sections with lines $1 a$ and $1 b$, taking advantage of the natural conduction block of the aortic and mitral annuli. The right atrium was further divided with lines 1 and 2 , such that the area paced (marked by an asterisk) was progressively smaller after each iteration. On the left subdivision, line 2 removed the area of the left atrial appendage, and a final line (3) subdivided the remaining tissue area around the pacing electrode. SVC, Superior vena cava; $I V C$, inferior vena cava.

line to the mitral annulus. A second ablation line extending superiorly from the pulmonary veins, above the roof of the left atrium, and down to the aortic annulus was created. Because the aortic annulus and mitral annulus do not conduct electrical impulses, this effectively partitioned the atria into 2 roughly equal segments. Subsequent ablations progressively excluded more atrial surface area from the subdivisions containing the pacing electrodes used for induction of AF. On the right atrium, ablation lines extended from beyond the cuff of atrial tissue on the superior and inferior venae cavae down onto the tricuspid valve annulus. Except for the ablations around the pulmonary veins and at the base of the left atrial appendage, each ablation was carried out with 1 jaw of the ablation device inserted through a purse-string suture into the atria.

After completion of the lesion set and the iterative approach to attempt induction of AF, the heart was arrested with concentrated potassium chloride solution. The aorta was cross-clamped, and the coronary circulation was infused with $60 \mathrm{~mL}$ of $1 \%$ 2,3,5-triphenyl-tetrazolium chloride solution. The heart was excised en bloc and then placed into 2,3,5-triphenyl-tetrazolium chloride solution and allowed to incubate for 45 minutes at room temperature to stain viable myocardium and aid in the visualization of the ablations lines. The atria were dissected free from the ventricle and all excess fat and connective tissue were removed. They were then digitally photographed next to a caliper set to $1 \mathrm{~cm}$ for calibration. Atrial surface area was calculated using commercial software (Adobe Photoshop, San Jose, Calif)

All data were expressed as mean \pm standard deviation. Analysis of variance with multiple comparisons was used for comparisons of more than 2 groups. Multiple comparisons were made with a post hoc test (Fisher's exact). A multivariable logistical regression was performed to develop a model for determining the probability of AF, as a function of conduction velocity, ERP, and surface area. Probability was assessed with SYSTAT software (SYSTAT Software Inc, Evanston, Ill).

\section{RESULTS}

\section{Acetylcholine Dose-Response Results}

There was a linear dose-response relationship between ERP and increasing doses of acetylcholine. These data are summarized in Figure 2. After 5 minutes of infusion, the ERP decreased to $83 \% \pm 10 \%, 69 \% \pm$
$10 \%, 60 \% \pm 12 \%$, and $57 \% \pm 26 \%$ of baseline at doses of $0.005,0.01,0.015$, and $0.02 \mathrm{mg} / \mathrm{Kg} / \mathrm{min}$, respectively. The mean ERP at baseline was $192 \pm$ $30 \mathrm{~ms}$. The response to acetylcholine was stable during the 20 minutes of infusion, and ERP returned to baseline within 5 minutes after the discontinuation of the acetylcholine infusion (Figure 3).

All animals survived the acute ablation set and were able to complete the entire study. As atrial surface area increased in each range of ERP, the probability of sustained AF increased (Figure 4). As ERP decreased in each range of area, the probability of sustained AF also increased.

A multivariable logistic regression analysis was performed to analyze the probability of AF. Atrial surface area and ERP were found to be statistically significant

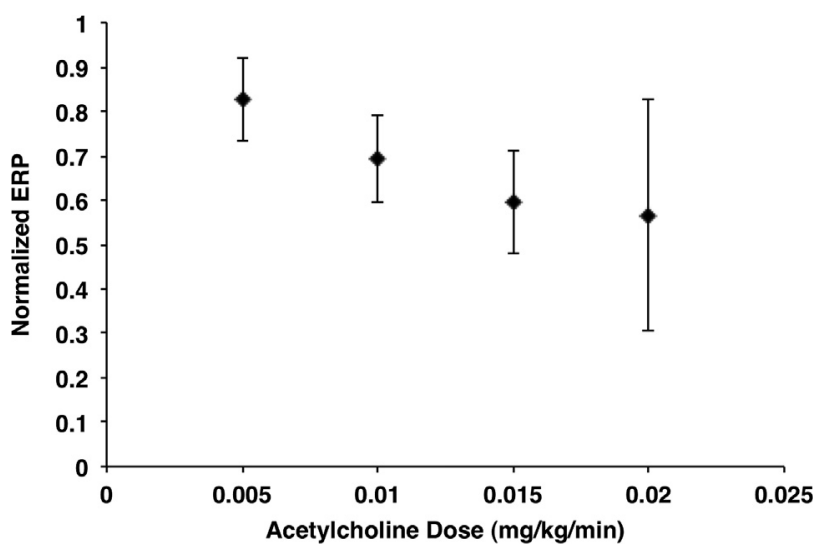

FIGURE 2. Dose-response relationship of normalized effective refractory period $(E R P)$ versus acetylcholine dose at peak effect. ERP was normalized to the ERP measured before the administration of acetylcholine. As the dose of acetylcholine increased, the measured ERP decreased $(P=.015)$. 


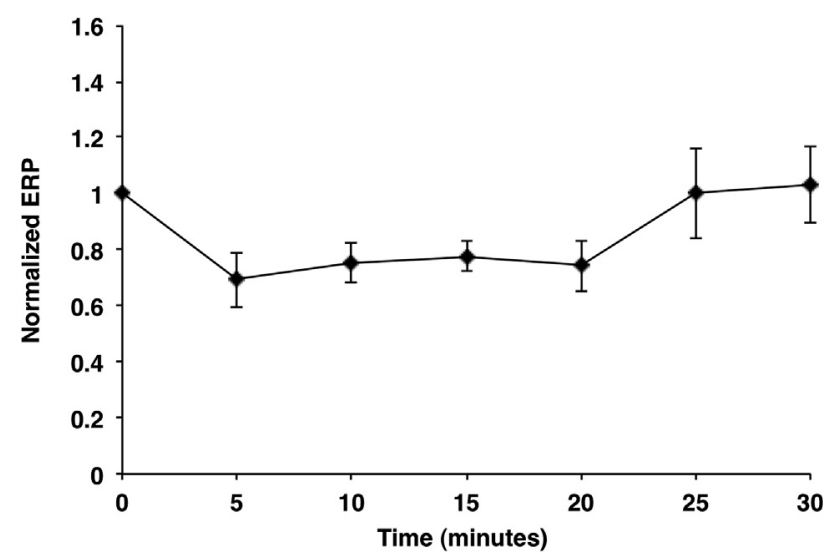

FIGURE 3. Time course of effective refractory period (ERP), normalized to initial value at time 0 before infusion of acetylcholine at $0.01 \mathrm{mg} / \mathrm{Kg} /$ min. After 5 minutes, the ERP decreased to a stable level. At time 20 minutes, the infusion was turned off, and ERP recovered to baseline level within 5 minutes.

factors affecting the probability of $\mathrm{AF}(P=.004$ and $P<.001$, respectively). Conduction velocity (average velocity $=1.03 \pm 0.17 \mathrm{~m} / \mathrm{s}$ ) was not a significant factor affecting the probability of AF in this study $(P=.668)$.

A multivariable logistic regression analysis was used to create a model for predicting the probability of sustained $\mathrm{AF}$ based on increasing surface area and decreasing ERP (Figure 5). This model was found to be highly predictive, with an area under the curve of the receiver-operator characteristic curve of 0.878 and a McFadden rho of 0.358 . A McFadden rho between 0.2 and 0.4 is considered satisfactory. ${ }^{16}$

The complete lesion set was sufficient to prevent sustained AF in all 9 animals. One ablation on each side was necessary to prevent sustained AF in $33 \%$ of the animals. Two ablations on each side were necessary to prevent

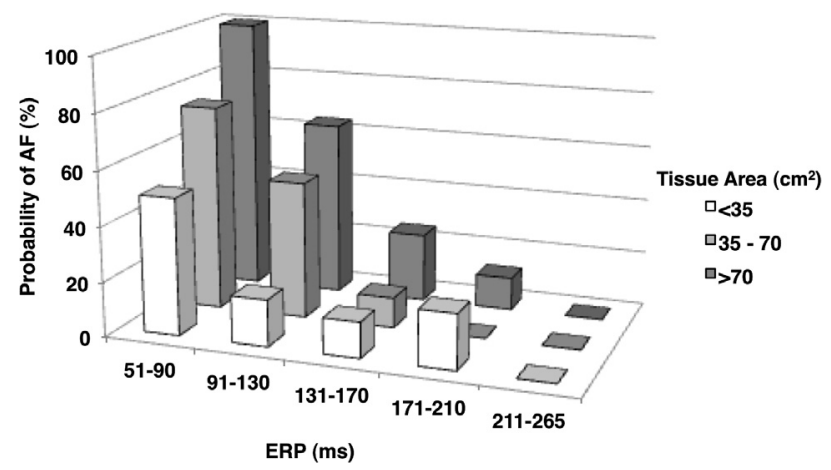

FIGURE 4. The probability of sustained atrial fibrillation $(A F)(y$-axis) is plotted against effective refractory period $(E R P)(x$-axis) and surface area (z-axis). As area decreased (dark grey to white), the probability of sustained $\mathrm{AF}$ decreased. As ERP increased, the probability of sustained AF decreased. sustained AF in $33 \%$ of animals, and all 3 right and left ablations were necessary in the last third of animals.

\section{DISCUSSION}

This study showed that the probability of sustained AF is dependent on increasing atrial surface areas and decreasing ERP. Reducing the surface area below a critical area prevented the atria from sustaining $\mathrm{AF}$ in this intact animal model. Previous work from this laboratory demonstrated this relationship in the in vitro setting, using an isolated canine atrial preparation. ${ }^{7}$ The translation of this model into the intact animal model represents a significant step toward applying the critical mass hypothesis to the clinical setting. It has been widely established that patients with large left atria have a higher rate of recurrent $\mathrm{AF}$ after the Cox maze procedure. ${ }^{12,17}$ Garrey $^{3}$ hypothesized that a critical mass of atrial tissue was necessary to sustain $\mathrm{AF}$ almost 75 years before the invention of the Cox maze procedure. Whereas clinical results have provided indirect support of his theory, this study provides direct evidence in support of the critical mass hypothesis in an in vivo model.

Although the mechanisms of $\mathrm{AF}$ remain incompletely understood, the findings in this study can help partially to explain the clinical results seen after more than 2 decades of surgical ablation. It has been established that patients with longstanding AF have higher rates of recurrence after the Cox maze procedure. ${ }^{17}$ In addition to having larger atria, patients with longstanding AF are likely to be older and to have slower conduction velocities and shorter refractory periods. It has been shown recently that patients with $\mathrm{AF}$ have increased fibrosis, resulting in a shortening of conduction velocities. ${ }^{18}$ In animal models, prolonged exposure to tachycardia leads to decreasing ERPs. ${ }^{19}$ These slower conduction velocities and shorter refractory periods yield shorter wavelengths, and the atrial surface area needed for sustained AF becomes smaller, as demonstrated in our intact model (Figure 4). Conduction velocity was not found to be a significant factor affecting the probability of AF in this study. This is likely due to the fact that conduction velocity was not varied pharmacologically by acetylcholine and that these normal animals had little physiologic variability in atrial conduction velocities. Conduction velocity throughout the experiment was consistently around the normal value of $1.0 \mathrm{~m} / \mathrm{s}(1.03 \pm 0.17 \mathrm{~m} / \mathrm{s})$.

The potential clinical application of these findings is significant. The logistic regression model derived from the data in this study was highly predictive for the probability of sustained AF if atrial surface area and ERP are known (area under the curve of receiver operating characteristic curve of 0.878). If these parameters were measured in a patient before receiving the Cox maze procedure, the likelihood of success of the procedure could be easily calculated. It is now possible to obtain these data on patients preoperatively, 


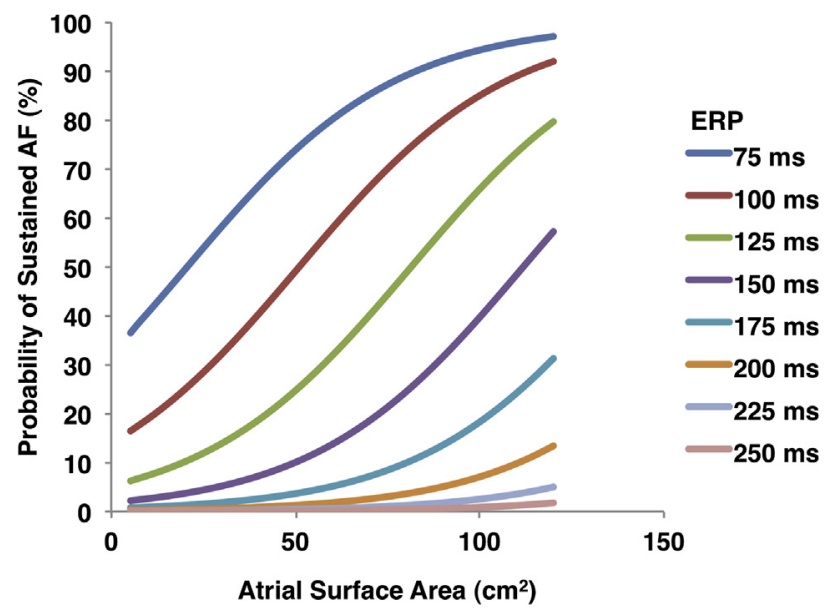

FIGURE 5. The predictive model of the sustainability of atrial fibrillation $(A F)$ is plotted. For each range of effective refractory period $(E R P)$, the probability of sustained AF (y-axis) increased with increasing surface area $(x$-axis). A line is plotted for specific values of ERP. As ERP increases, the probability of sustained AF decreases. The area under the curve of the receiver operating characteristic curve is 0.878 , and the McFadden rho ${ }^{2}$ was 0.358 .

noninvasively. Contrast-enhanced computed tomographic (CT) scans are capable of measuring atrial volumes and surface areas, and studies have demonstrated good correlation with the clinical standard of echocardiography. ${ }^{20}$ Using CT to determine atrial surface area preoperatively would not be technically more difficult than measuring volumes. The electrophysiologic parameters (ERP and conduction velocity) could be determined by cardiac mapping. This can be performed noninvasively using ECG imaging, which uses body surface potentials and anatomic data derived from CT scans to calculate the epicardial surface potentials of subjects noninvasively. ${ }^{21}$ Our group recently has published a clinical study of 36 patients with AF, establishing the accuracy of ECG imaging and its ability to define activation patterns in $\mathrm{AF}^{22}$ In patients with $\mathrm{AF}$, conduction velocity could be measured with ECG imaging, and local ERP could be estimated using minimal AF intervals using the technique described in this study. ${ }^{14}$

On the basis of the observation that larger atria had higher rates of failure, one strategy to increase the success of the Cox maze procedures has been to excise tissue to reduce the size of the left atrium. ${ }^{23}$ Romano and colleagues ${ }^{23}$ have reported an $89 \%$ success rate with this technique, but this has not been proven in a randomized clinical trial. The logistic regression model from this study could be used to determine whether or not atrial reduction would be necessary. Moreover, ECG imaging and CT could be used to determine which patients would not benefit from an operation, because their critical area might be too small to practically achieve with ablation techniques or the number of lesions could severely limit atrial contractility. For instance, this could be the case in patients with severe atrial fibrosis and very slow conduction velocities.

\section{Limitations}

A more complete model for the sustainability of AF will require manipulation of the conduction velocity independently from ERP to better define the importance of conduction velocity as it relates to wavelength. The study was conducted in healthy, young pigs. Before application to the clinical setting, an ideal model would involve diseased tissue. However, it is likely that the basic substrates of atrial surface area, ERP, and conduction velocity will remain the important factors in determining the probability of sustaining AF even in diseased atrial tissue.

Finally, the critical mass hypothesis was based on a multiple wavelet theory of AF. It is now clear that AF can originate from focal sources that may be the result of triggered activity or a small single reentrant circuit (rotor) with fibrillatory conduction in addition to multiple wavelets. Vaidya and associates ${ }^{24}$ demonstrated ventricular fibrillation driven by very small reentrant circuits with wavelengths of $16.8 \mathrm{~mm}$ in the mouse heart. ${ }^{24}$ The conduction velocities measured in their study were an order of magnitude smaller than the ones seen in this study and achieved on isolated Langendorff preparations. It has also been shown that a small, less than $1-\mathrm{cm}$ single stable reentrant circuit can drive $\mathrm{AF}$ in atrial tissue in the $\operatorname{dog}{ }^{25}$ However, the ERP in that case was extremely short $(<50 \mathrm{~ms})$. It is not clear that these very small rotors will remain stationary over an extended period of time. The porcine model used in this in vivo study is likely more applicable to the clinical setting.

Computer simulations of atrial tissue by Kneller and colleagues ${ }^{26}$ also demonstrated fibrillatory conduction away from a single stable reentrant circuit. In this computer simulation, acetylcholine stabilized the driving rotor while promoting fibrillatory conduction in wavelets through the rest of the tissue. However, in this same model Zou and associates ${ }^{5}$ showed that by changing the distribution and concentration of acetylcholine, either a single rotor or multiple reentrant rotors could maintain fibrillatory activity in their model and the duration of AF was dependent on tissue size. ${ }^{5} \mathrm{In}$ another simulation, $\mathrm{Qu}^{6}$ demonstrated that fibrillation duration increased exponentially with tissue area and also increased exponentially with area to perimeter ratio. In small areas of atrial tissue where the area is small relative to the perimeter, these rotors are more likely to collide with the edge of the tissue and terminate. The ablation lines in the Cox maze procedure and ablation lines used in this study not only decrease the area, but also decrease the area/perimeter ratio.

The present study supports the critical mass hypothesis by defining a relationship between atrial surface area, ERP, and the probability of sustained AF. In the future, being able to determine critical surface area could be used 
to tailor surgical ablation to the individual patient's own atrial anatomy and electrophysiology.

We gratefully acknowledge the technical assistance of Diane Toeniskoetter and Naomi Still.

\section{References}

1. Lloyd-Jones D, Adams R, Carnethon M, De Simone G, Ferguson TB, Flegal K, et al. Heart disease and stroke statistics-2009 update: a report from the American Heart Association Statistics Committee and Stroke Statistics Subcommittee. Circulation. 2009;119:e21-181.

2. Vidaillet H, Granada JF, Chyou PH, Maassen K, Ortiz M, Pulido JN, et al. A population-based study of mortality among patients with atrial fibrillation or flutter. Am J Med. 2002;113:365-70.

3. Garrey W. The nature of fibrillary contraction of the heart: its relation to tissue mass and form. Am J Physiol. 1914;33:397-414.

4. Weiner N, Rosenbluth A. The mathematical formulation of the problem of conduction of impulses in a network of connected excitable elements, specifically in cardiac muscle. Arch Inst Cardiol Mex. 1946;16:205-65.

5. Zou R, Kneller J, Leon LJ, Nattel S. Substrate size as a determinant of fibrillatory activity maintenance in a mathematical model of canine atrium. Am J Physiol Heart Circ Physiol. 2005;289:H1002-12.

6. Qu Z. Critical mass hypothesis revisited: role of dynamical wave stability in spontaneous termination of cardiac fibrillation. Am J Physiol Heart Circ Physiol. 2006;290:H255-63.

7. Byrd GD, Prasad SM, Ripplinger CM, Cassilly TR, Schuessler RB, Boineau JP, et al. Importance of geometry and refractory period in sustaining atrial fibrillation: testing the critical mass hypothesis. Circulation. 2005; 112(9 Suppl):I7-13.

8. Haissaguerre M, Jais P, Shah DC, Takahashi A, Hocini M, Quiniou G, et al. Spontaneous initiation of atrial fibrillation by ectopic beats originating in the pulmonary veins. N Engl J Med. 1998;339:659-66.

9. Calkins H, Brugada J, Packer DL, Cappato R, Chen SA, Crijns HJ, et al. HRS/ EHRA/ECAS expert consensus statement on catheter and surgical ablation of atrial fibrillation: recommendations for personnel, policy, procedures and follow-up. A report of the Heart Rhythm Society (HRS) Task Force on catheter and surgical ablation of atrial fibrillation. Heart Rhythm. 2007;4:816-61.

10. Cox JL, Schuessler RB, Boineau JP. The development of the Maze procedure for the treatment of atrial fibrillation. Semin Thorac Cardiovasc Surg. 2000;12:2-14.

11. Voeller RK, Bailey MS, Zierer A, Lall SC, Sakamoto S, Aubuchon K, et al. Isolating the entire posterior left atrium improves surgical outcomes after the Cox maze procedure. J Thorac Cardiovasc Surg. 2008;135:870-7.
12. Kosakai Y. Treatment of atrial fibrillation using the Maze procedure: the Japanese experience. Semin Thorac Cardiovasc Surg. 2000;12:44-52.

13. Farges JP, Faucon G, Lievre M, Ollagnier M. Relationship between atrial and ventricular rates of fibrillation and cardiac contractile tissue effective refractory periods in the dog. Br J Pharmacol. 1978;63:587-91.

14. Kim KB, Rodefeld MD, Schuessler RB, Cox JL, Boineau JP. Relationship between local atrial fibrillation interval and refractory period in the isolated canine atrium. Circulation. 1996;94:2961-7.

15. Voeller RK, Zierer A, Lall SC, Sakamoto S, Schuessler RB, Damiano RJ Jr. Efficacy of a novel bipolar radiofrequency ablation device on the beating heart for atrial fibrillation ablation: a long-term porcine study. $J$ Thorac Cardiovasc Surg. 2010;140:203-8.

16. Hensher D, Johnson LW. Applied discrete choice modeling. London (United Kingdom): Croom Helm; 1981.

17. Gaynor SL, Schuessler RB, Bailey MS, Ishii Y, Boineau JP, Gleva MJ, et al. Surgical treatment of atrial fibrillation: predictors of late recurrence. $J$ Thorac Cardiovasc Surg. 2005;129:104-11.

18. Swartz MF, Fink GW, Lutz CJ, Taffet SM, Berenfeld O, Vikstrom KL, et al. Left versus right atrial difference in dominant frequency, $\mathrm{K}(+)$ channel transcripts, and fibrosis in patients developing atrial fibrillation after cardiac surgery. Heart Rhythm. 2009;6:1415-22.

19. Gaspo R, Bosch RF, Talajic M, Nattel S. Functional mechanisms underlying tachycardia-induced sustained atrial fibrillation in a chronic dog model. Circulation. 1997;96:4027-35.

20. Christiaens L, Lequeux B, Ardilouze P, Ragot S, Mergy J, Herpin D, et al. A new method for measurement of left atrial volumes using 64-slice spiral computed tomography: comparison with two-dimensional echocardiographic techniques. Int J Cardiol. 2009;131:217-24.

21. Rudy Y. Noninvasive imaging of cardiac electrophysiology and arrhythmia. Ann N Y Acad Sci. 2010;1188:214-21.

22. Cuculich PS, Wang Y, Lindsay BD, Faddis MN, Schuessler RB, Damiano RJ Jr, et al. Noninvasive characterization of epicardial activation in humans with diverse atrial fibrillation patterns. Circulation. 2010;122:1364-72.

23. Romano MA, Bach DS, Pagani FD, Prager RL, Deeb GM, Bolling SF. Atrial reduction plasty Cox maze procedure: extended indications for atrial fibrillation surgery. Ann Thorac Surg. 2004;77:1282-7; discussion 1287.

24. Vaidya D, Morley GE, Samie FH, Jalife J. Reentry and fibrillation in the mouse heart. A challenge to the critical mass hypothesis. Circ Res. 1999;85:174-81.

25. Schuessler RB, Grayson TM, Bromberg BI, Cox JL, Boineau JP. Cholinergically mediated tachyarrhythmias induced by a single extrastimulus in the isolated canine right atrium. Circ Res. 1992;71:1254-67.

26. Kneller J, Zou R, Vigmond EJ, Wang Z, Leon LJ, Nattel S. Cholinergic atrial fibrillation in a computer model of a two-dimensional sheet of canine atrial cells with realistic ionic properties. Circ Res. 2002;90:E73-87. 